\title{
Modified Chest X-Ray Scoring System in Evaluating Severity of COVID-19 Patient in Dr. Soetomo General Hospital Surabaya, Indonesia
}

\author{
Rosy Setiawati (D) \\ Anita Widyoningroem (1D \\ Triwulan Handarini (D) \\ Fierly Hayati $\mathbb{D}^{\prime}$ \\ Agnes Triana Basja (D) \\ Atrikha Rahma Dyana Surya \\ Putri (iD) ${ }^{1}$ \\ Merlin Guntur Jaya $\mathbb{D}^{2}$ \\ Jessica Andriani (iD) ${ }^{2}$ \\ Melina Rosita Tanadi (iD ${ }^{2}$ \\ Imran Harsam Kamal (iD) ${ }^{2}$ \\ 'Department of Radiology, Faculty of \\ Medicine, Universitas Airlangga - Dr. \\ Soetomo General Hospital Surabaya, \\ Surabaya, Indonesia; ${ }^{2}$ Faculty of Medicine \\ Universitas Airlangga, Surabaya, Indonesia
}

Correspondence: Rosy Setiawati 60286

Tel +62 87853300019

Email rosy-s@fk.unair.ac.id
Introduction: The management of COVID-19 patients requires efficiency and accuracy in methods of detection, identification, monitoring, and treatment feasible in every hospital. Aside from clinical presentations and laboratory markers, chest x-ray imaging could also detect pneumonia caused by COVID-19. It is also a fast, simple, cheap, and safe modality used for the management of COVID-19 patients. Established scoring systems of COVID-19 chest x-ray imaging include Radiographic Assessment of Lung Edema (RALE) and Brixia classification. A modified scoring system has been adopted from BRIXIA and RALE scoring systems and has been made to adjust the scoring system needs at Dr. Soetomo General Hospital, Indonesia. This study aims to determine the value of scoring systems through chest $\mathrm{x}$-ray imaging in evaluating the severity of COVID-19.

Methods: Data were collected from May to June of 2020 who underwent chest x-ray evaluation. Each image is then scored using three types of classifications: modified score, RALE score, and Brixia score. The scores are then analyzed and compared with the clinical conditions and laboratory markers to determine their value in evaluating the severity of COVID-19 infection in patients.

Results: A total of 115 patients were males (51.1\%) and 110 were females (48.9\%). All three scoring systems are significantly correlated with the clinical severity of the disease, with the strengths of correlation in order from the strongest to weakest as Brixia score $(p<0.01$, correlation coefficient 0.232$)$, RALE score ( $p<0.01$, correlation coefficient 0.209$)$, and Dr. Soetomo General Hospital score $(p<0.01$, correlation coefficient 0.194). All three scoring systems correlate significantly with each other. Dr. Soetomo General Hospital score correlates more towards Brixia score $(\mathrm{p}<0.01$, correlation coefficient 0.865$)$ than RALE score $(\mathrm{p}<0.01$, correlation coefficient 0.855 ). Brixia to RALE score correlates with a coefficient of $0.857(\mathrm{p}<0.01)$.

Conclusion: The modified scoring system can help determine the severity of the disease progression in COVID-19 patients especially in areas with shortages of facilities and specialists.

Keywords: Brixia score, chest radiographs, COVID-19, modified score, RALE score

\section{Introduction}

COVID-19 appeared in Wuhan, China on the late December 19 with fever, cough, and myalgia as the most common symptoms. ${ }^{1}$ The disease manifests in varying degrees from mild to severe and may progress to acute respiratory distress syndrome (ARDS). ${ }^{2}$ In current cases, the fatality rate of COVID-19 varies between countries, although the exact number of mortality rate remains unknown, due to the 
number of asymptomatic cases. ${ }^{3}$ Within only a few months, the pathogen had spread all over the world and contributed to a $3-5 \%$ of mortality. ${ }^{4}$ By the end of March 2020, the Case Fatality Rate (CFR) of COVID-19 in Indonesia was $8.9 \%$. This is higher than the mean CFR all over the world. ${ }^{5}$ Radiological examination is reported to play an important role in the diagnosis of COVID-19. A systematic review of case series with a total of 919 patients in China and Korea suggested that chest X-ray (CXR) may not be reliable for diagnosing COVID-19 in its early stage. Only in its intermediate and severe stages is when COVID-19's feature of the lungs is seen on CXR. The British Society of Thoracic Imaging (BSTI) suggested chest radiography to all seriously ill patients (oxygen saturation $<94 \%$, National early warning score (NEWS) $>3$ ) and if "clinically required". 6 On the other hand, chest computed tomography $(\mathrm{CT})$ could provide a more accurate identification of severe lung diseases in COVID-19 with a sensitivity of $83.3 \%$ and a specificity of $94 \%{ }^{7}$ Although chest-CT is more sensitive and specific than CXR, interpreting CXRs had always been a routine practice for clinicians to rule out other causes of respiratory disease. ${ }^{8}$ The lack of CT-scans and radiologists, especially in developing countries such as Indonesia, makes CXR a great substitute to diagnose and determine the severity and progression of lung abnormalities in COVID-19 patients. ${ }^{9}$

The currently established CXR scoring systems are Brixia and Radiographic Assessment of Lung Edema (RALE) scoring systems. Brixia scoring system has been widely used as a tool to monitor the severity and progression of COVID-19 pneumonia in Tongji Hospital, Wuhan and Azienda Socio Sanitaria Territoriale, Spedali Civili of Brescia, Italy. ${ }^{10}$ RALE scoring system, presented by Wong et al, has been used in Queen Mary Hospital, Hong Kong; Pamela Youde Nethersole Eastern Hospital, Hong Kong; The University of Hong Kong; Shenzhen Hospital, Shenzhen; and University Hospital Careggi. ${ }^{11-13}$ One of the important differences between the two scorings is that RALE can be done by a general practitioner due to its simplicity, while Brixia is designed to be used by a trained radiologist. The RALE scoring system only divides the lung into two regions, making it not specific to smaller lesions. $^{14}$

Seeing the current conditions regarding the lack of distribution of trained radiographers and radiologists in Indonesia, we proposed a CXR scoring system for evaluating the severity of COVID-19 patients in Dr. Soetomo General Hospital, Surabaya, Indonesia. A modified scoring system has been adopted from Brixia and RALE scoring systems, and has been made to adjust to the needs and resources of Dr. Soetomo General Hospital Surabaya, Indonesia. This scoring system is aimed to be applicable to mild pneumonia cases and provide a faster and more accurate analysis. This study aims to determine the value of scoring systems through CXR imaging in evaluating the severity of COVID-19.

\section{Materials and Methods}

This is a retrospective study of patients with RT-PCRconfirmed COVID-19 in Dr. Soetomo General Hospital. This study was conducted in accordance with the Declaration of Helsinki. This study was approved by the Medical Research Ethics Committee of Dr. Soetomo General Hospital, Surabaya. All participants included had given their written informed consent to participate in this study during admission. In cases of decreased consciousness and severe illness, written informed consent was represented by next of kin.

We included 225 patients who came to the emergency ward and outpatient clinic from May - June 2020. Patients with symptoms and who had RT-PCR-confirmed COVID19 and positive CXR abnormalities were included. In this study, we analyzed the initial CXR of when the patient was first admitted. The CXRs were analyzed by 2 radiologists with an experience of more than 10 years.

Chest radiographs were acquired following the usual protocols and performed with posteroanterior or anteroposterior projection. The radiographs were then interpreted as normal or abnormal. The abnormalities are described by the distribution (upper zone predominant, middle zone predominant, or lower zone predominant), side (right or left lung), and peripheral or perihilar dominance. Other features, such as pleural effusions and nodules, will also be mentioned.

The severity based on the imaging was calculated using Brixia, RALE, and the Dr. Soetomo General Hospital scoring system (Modified Chest X-ray Scoring System).

Brixia scoring system divided the lungs into six regions. There were 2 lines dividing the lungs into six regions; the first line was drawn at the level of the inferior wall of the aortic arch and the second line at the level of the right inferior pulmonary vein. Radiologists rate each region from 0 to 3 based on the severity of the lesion. Score 0 for no lung abnormalities, 1 for interstitial infiltrates, 2 for interstitial and alveolar infiltrates (interstitial dominant), and 3 for interstitial and alveolar infiltrates (alveolar 
dominant). The score ranges from 0 to 18 . Other findings, such as pleural effusion and pulmonary vessel enlargements, were not included in Brixia scoring system. ${ }^{10}$

The RALE scoring system divided the lungs into 2 regions, left and right lung. Each lung is scored from 0 to 4 each; score 0 for no involvement, 1 for less than $25 \%$ involvement, 2 for $25 \%-50 \%$ involvement, 3 for $50-75 \%$ involvement, and score 4 for more than $75 \%$ involvement. The maximum score for RALE scoring is $8 .{ }^{14}$

The Modified Chest X-ray Scoring System calculated the score or severity from the posteroanterior and anterior projection of CXR by dividing the lungs into 6 regions. Two lines divided the lung horizontally, resulting in each lung into having 3 regions, as shown in (Figure 1). Each region was rated 0 to 2 based on the lesions; score 0 if there is no involvement, 1 if infiltrates or consolidations are less than $50 \%$, and 2 if infiltrates or consolidations are more than $50 \%$. The maximum score for the Modified Chest X-ray scoring system is 12 . The final scores were then classified further into mild (score 1-4), moderate (score 5-8) and severe (score 9-12). The example of the application of this scoring system is shown in (Figure 2).

The data were analyzed with IBM SPSS version 25 software. Kolmogorov-Smirnov test was used to assess the normality of the distribution of each scoring system. Spearman correlation test was used to correlate Modified Chest X-Ray scoring system with RALE and Brixia

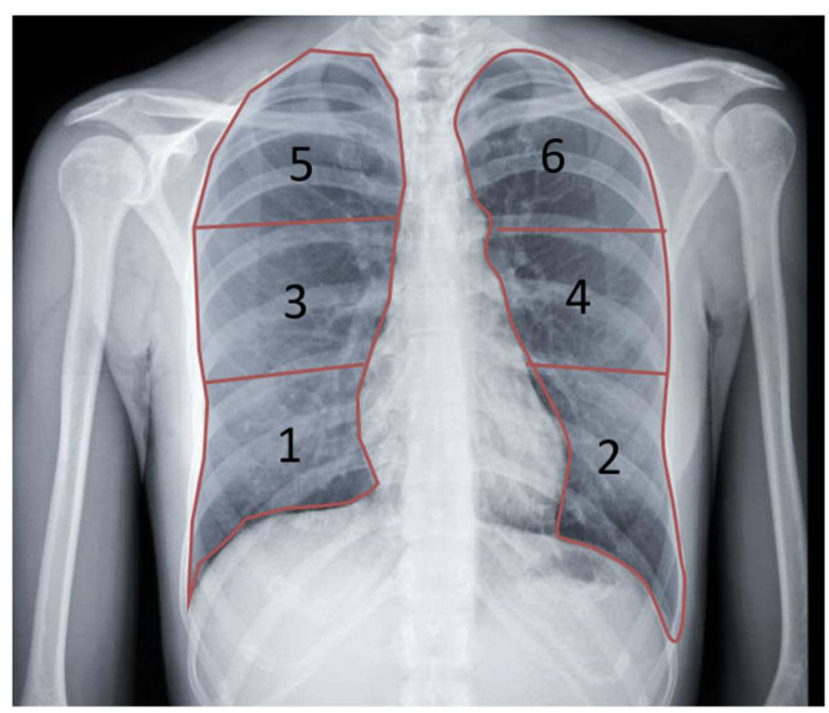

Figure I Lung field division using Modified Chest X-ray Scoring System on chest posteroanterior (PA) projection, The lung field is divided into six zones lower zone ( 1 or 2 ) is under the inferior wall of the lower right pulmonary vein (lung base), middle zones ( 3 or 4 ) is below the inferior wall of the aortic arch and above the inferior wall of the lower right pulmonary vein (ie, hilar structures), and upper zone (5 or 6) is above the inferior wall of the aortic arch. scoring system. $\mathrm{P}$ value of $<0.01$ was considered statistically significant.

\section{Results}

\section{Patient Characteristics}

The characteristics of patients were summarized in (Table 1). This study included 115 men (51.1\%) and 110 women (48.9\%), with a mean age of 53 (21-81) years. Most presented with mild pneumonia $(29.8 \%)$, and no comorbidities $(51.1 \%)$.

\section{Chest Radiography Features}

Out of 225 patients, 210 patients had abnormal chest radiograph findings as shown in (Table 2). The distribution of lesions was mostly upper, middle and lower zone predominance (103 of $225 ; 45.6 \%$ ). A total of 179 out of 225 (79.2\%) presented with lesions on bilateral lungs, and 121 out of $225(53.5 \%)$ were peripheral dominant. Other features of CXR included pleural effusion in 28 patients $(12.4 \%)$ and pulmonary nodules in 33 patients (14.6\%).

\section{Chest Radiography Scoring and Clinical Feature}

Based on the RALE scores, most patients of all clinical characteristics scored 5 (17.3\%). Asymptomatic patients and those with mild pneumonia mostly scored 2 (11 patients, $4.9 \%$, and 17 patients, $7.6 \%$ ). Patients with severe pneumonia mostly scored in 5 (9 patients, 4\%), while those with ARDS mostly scored 4 and 5 (4 patients, $1.8 \%$ ). Septic patients mostly scored 7 (7 patients, $3.1 \%$ ), while patients with septic shock mostly scored 5 (7 patients, 3.1\%).

On the other hand, the Brixia scores ranged from 0 to 18. Most patients scored 6 (22 patients, 9.8\%). Asymptomatic patients scored 6 (11 patients, 4.9\%), while most of the patients with mild pneumonia scored 5 (8 patients, $3.6 \%$ ); those with severe pneumonia scored 7 (7 patients, 3.1\%); those with ARDS patients scored 6; 15, and 18 ( 2 patients, $0.9 \%$ ); septic patients scored 6 (5 patients, 2.2\%); and septic shock patients scored 14 (6 patients, $2.7 \%$ ).

The Modified Chest X-ray score ranged from 0 to 12 . Most patients scored in 6 (30 patients, 13.3\%), while there were no patients who scored 13-17. Asymptomatic patients mostly scored in 6 (11 patients, 4.9\%); patients with mild pneumonia mostly scored in 9 (11 patients, $4.9 \%$ ); patients with severe pneumonia mostly scored in 


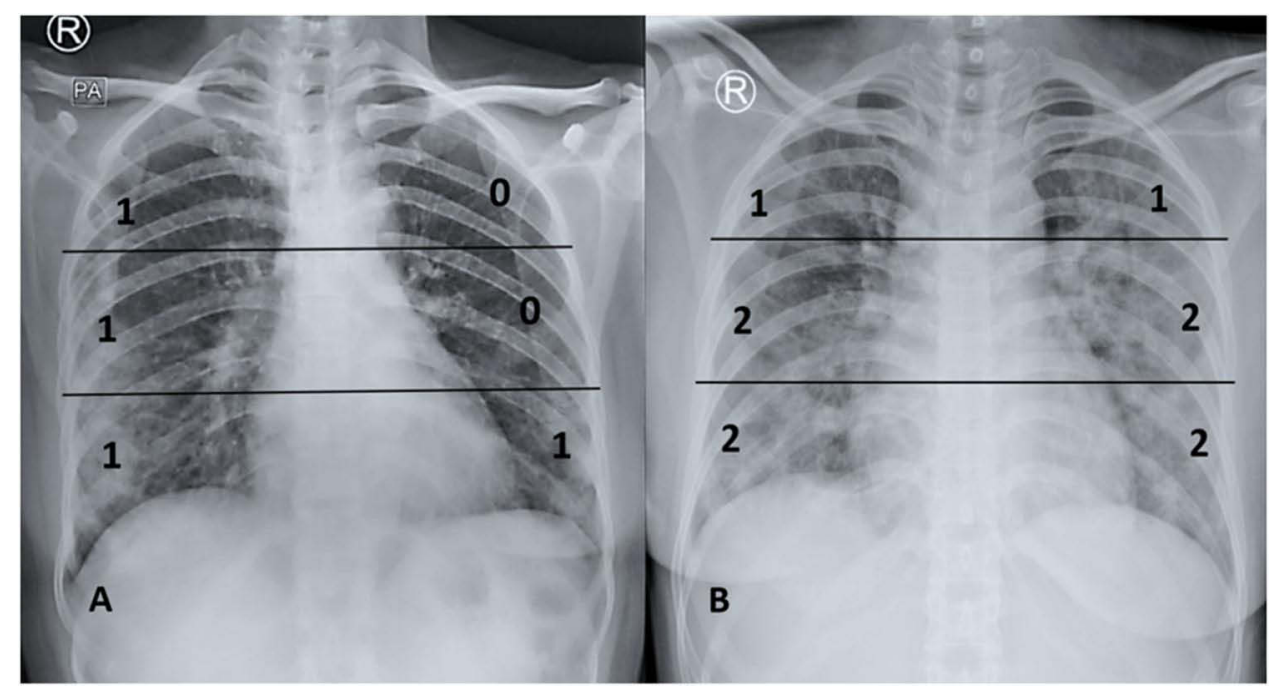

Figure 2 Two case illustrations of applying Modified Chest X-ray Scoring System on AP projection. (A). The total calculated score of the 6 chest divisions is 4, classified as mild severity score. (B) The total calculated score of the 6 chest divisions is 9 , classified as severe severity score.

6 (10 patients, 4.4\%). ARDS patients scored in 7 (4 patients, 1.8\%); septic patients mostly scored in 5 (6 patients, $2.7 \%$ ); while patients with septic shock mostly scored in 10 (7 patients, 3.1\%).

Based on (Table 3), most patients were classified as severe based on the CXR score classification (92 patients, 40.89\%). Most patients with mild CXR score classification also presented with mild clinical presentation (19 patients, $44.19 \%$ ). Those classified as moderate were mostly asymptomatic and had a clinical feature of mild pneumonia (23 patients, $25.5 \%$ ), while those classified as severe in

Table I Sample Characteristic at Presentation

\begin{tabular}{|l|l|}
\hline Parameter & $\begin{array}{l}\text { No. of Patients } \\
(\mathbf{n}=\mathbf{2 2 5})\end{array}$ \\
\hline $\begin{array}{l}\text { Patient Characteristic } \\
\text { Male }\end{array}$ & $115(51.1 \%)$ \\
Female & $110(48.9 \%)$ \\
Age (year) & $53 \pm 13$ \\
\hline Comorbidities & \\
No comorbid & $115(51.1 \%)$ \\
Hypertension & $10(4.4 \%)$ \\
Obesity & $4(1.8 \%)$ \\
Diabetes & $17(7.6 \%)$ \\
Heart abnormalities & $5(5 \%)$ \\
Other lung abnormalities & $6(2.7 \%)$ \\
Renal abnormalities & $4(1.8 \%)$ \\
Hepatitis & $9(4.0 \%)$ \\
Combine comorbid & $40(6.7 \%)$ \\
Others & $15(6.5 \%)$ \\
\hline
\end{tabular}

CXR classification mostly had mild pneumonia as a clinical feature (25 patients, $27.17 \%$ )

\section{Correlation of Chest Radiography Scoring Categorized and Clinical Feature}

The correlation coefficient of the CXR was 0.164 $(\mathrm{p}<0.01)$.

Table 2 Radiographic Findings on Chest Radiographs

\begin{tabular}{|l|l|}
\hline Radiographic Characteristic & $\begin{array}{l}\text { No. of Findings } \\
\text { (n = 225) }\end{array}$ \\
\hline Normal chest radiograph & $15(6.6 \%)$ \\
\hline Distribution of lesion & \\
Upper Zone predominant & $1(0.4 \%)$ \\
Middle Zone predominant & $4(1.8 \%)$ \\
Lower Zone predominant & $33(14.6 \%)$ \\
Upper and Middle zone predominant & $3(1.3 \%)$ \\
Upper and Lower zone predominant & $1(0.4 \%)$ \\
Middle and Lower zone predominant & $65(28.8 \%)$ \\
Upper, Middle, and Lower zone predominant & $103(45.6 \%)$ \\
Right Lung & $14(6.2 \%)$ \\
Left Lung & $17(7.5 \%)$ \\
Bilateral Lung & $179(79.2 \%)$ \\
Peripheral predominant & $121(53.5 \%)$ \\
Perihilar predominant & $5(2.2 \%)$ \\
Peripheral and perihilar predominant & $84(37.2 \%)$ \\
\hline Lung Abormality Features on chest x-ray & \\
Reticular-nodular opacities & $33(11.6 \%)$ \\
Patchy opacities & $35(12.4 \%)$ \\
Consolidation & $187(66.1 \%)$ \\
Pleural effusion & $28(9.8 \%)$ \\
\hline
\end{tabular}


Table 3 Modified Chest X-Ray Scores and Clinical Feature Classification

\begin{tabular}{|l|l|l|l|l|l|l|l|}
\hline & Asymptomatic & Mild Pneumonia & Severe Pneumonia & ARDS & Sepsis & Septic Shock & Total \\
\hline Mild & 8 & 19 & 5 & 3 & 4 & 4 & 43 \\
Moderate & 23 & 23 & 14 & 7 & 14 & 9 & 90 \\
Severe & 11 & 25 & 19 & 5 & 15 & 17 & 92 \\
\hline
\end{tabular}

Table 4 Analysis Result Spearman Correlation of Modified Chest X-Ray, Brixia and RALE Scores

\begin{tabular}{|l|l|l|}
\hline & Brixia Score & RALE Score \\
\hline Modified Chest-xray Score & $r=0.865$ & $r=0.855$ \\
& $P<0.001$ & $P<0.001$ \\
& $n=225$ & $n=225$ \\
\hline RALE Score & $r=0.802$ & \\
& $P<0.001$ & \\
& $n=225$ & \\
\hline
\end{tabular}

\section{Chest Radiography Scoring Correlation with Each Other}

The correlation coefficient between the Modified chest $\mathrm{X}$-Ray score and Brixia score was $0.865(\mathrm{p}<0.01)$, and the correlation coefficient of the Modified chest X-Ray score and RALE score was $0.855(p<0.01)$ (Table 4). On the other hand, the correlation coefficient of Brixia and RALE score was slightly lower, which was 0.802 $(\mathrm{p}<0.01)$. This indicated that the Modified Chest X-ray Scoring System had a higher correlation coefficient relative to both established Brixia and RALE scoring systems.

\section{Discussion}

In this pandemic, an accurate radiological approach is necessary for a more rapid classification of COVID-19 patients. CXR may not be as sensitive as CT, but it still plays a major role in developing countries that lack more sophisticated modalities. Moreover, CXR can be brought to the patient's bedside, minimizing the risk of crossinfections. ${ }^{15}$ Although CXR has a low sensitivity to earlystage disease of COVID-19, it can be used for monitoring the advancement and preceding stages of COVID-19 especially in critical care. ${ }^{16}$

Signorino et al stated that Brixia score system to be a good scoring system that provided detailed presentation and distribution of the lung (6 regions) and a sensibility (4 levels). ${ }^{17}$ Borghesi et al also stated that Brixia scoring is a very useful method for ranking the stratification risk of patients with COVID-19 infection based on the severity of cases. ${ }^{10}$ However, Brixia scoring requires a trained radiologist to interpret a CXR, due to its more complex indicators and nature for diagnosing COVID-19 pneumonia. This could be an obstacle for primary healthcare physicians who require an accurate and simple technique for early diagnosis of COVID-19 pneumonia, but lack the knowledge and experience to do so. Meanwhile, RALE scoring can predict supplemental oxygen requirements and the need for ICU admission for mechanical ventilation. ${ }^{15,18}$ Compared to Brixia, RALE scoring lacks a detailed and more complex indicator in scoring chest $\mathrm{x}$-rays to diagnose COVID-19 pneumonia. We introduced the use of the Modified Chest X-ray Scoring System at Dr. Soetomo Academic General Hospital and compared it with the established Brixia and RALE scoring systems.

In this study, we found that most of the patients with severe clinical presentation (severe pneumonia, ARDS, sepsis, and septic shock) had 5 or more score with RALE scoring system. Meanwhile, patients with severe clinical presentation had 6 or more score with the Brixia scoring. The Modified Chest X-Ray scoring system showed 5 or more score for patients with severe clinical presentation.

All three scoring systems correlated significantly with one another. Modified Chest X-ray Scoring System of Dr. Soetomo General Hospital correlates more towards Brixia $(p<0.01$, correlation coefficient 0.865) than RALE $(p<0.01$, correlation coefficient 0.855$)$. This result indicates the reliability of Modified Chest X-ray Scoring System in diagnosing COVID-19 pneumonia, and can help in the determination of management of COVID-19 patients early on. However, it should also be noted that COVID-19 patients do not always present with pneumonia.

Modified Chest X-ray Scoring System of Dr. Soetomo General Hospital helps medical professionals in diagnosing COVID-19 pneumonia through a simple and fast method. Further study regarding its efficacy on a larger scale and possibly more heterogeneous cases and should be performed in the hope of its implementation on multiple healthcare center with X-ray devices and without CT scans. Also, study of the correlation of the scoring and the 
outcome of care with serial radiographs will add great value to predict patient condition, monitoring progression, later stages of COVID-19, and better management of the disease.

The main limitation of this study is the lack of comparison between the scoring systems and patient's clinical condition because some clinical data were only available in a small percentage of subjects. Another limitation of this study is the lack of comparison between chest x-ray score and patient comorbidities (ie, hypertension, diabetes, cardiovascular disease, and oncologic history) or final outcome.

\section{Conclusion}

Chest radiography can be considered as a tool to diagnose or measure the severity of COVID-19 with certain approach through scoring systems such as Brixia Score, RALE Score, and the Modified Chest X-Ray score of Dr. Soetomo General Hospital, although it may still be less sensitive than CT-Scan. The modified scoring system can help determine COVID-19 patient's severity, especially in areas with shortages of resources and specialists.

\section{Acknowledgments}

A sincere gratitude to all my colleagues, family, and all the patients included in this study.

\section{Disclosure}

The authors report no conflicts of interest in this work.

\section{References}

1. Zhu N, Zhang D, Wang W, et al. A novel coronavirus from patients with pneumonia in China, 2019. $N$ Engl J Med. 2020;382(8):727-733. doi:10.1056/NEJMoa2001017

2. Singhal TA. Review of Coronavirus Disease-2019 (COVID-19). Indian J Pediatr. 2020;87(4):281-286. doi:10.1007/s12098-02003263-6

3. Roser M, Ritchie H, Ortiz-Ospina E. Coronavirus @ Ourworldindata. Org [Internet]. 2020. Available from: https://ourworldindata.org/coro navirus. Accessed May 13, 2021.
4. Volpert V, Banerjee M, D’Onofrio A, Lipniacki T, Petrovskii S, Tran VC. Coronavirus - Scientific insights and societal aspects. Math Model Nat Phenom. 2020;15:1-8. doi:10.1051/mmnp/2020010

5. Setiati S, Azwar MK. COVID-19 and Indonesia. Acta medica Indonesiana. 2020;52(1):84-89.

6. Cleverley J, Piper J, Jones MM. The role of chest radiography in confirming covid-19 pneumonia. BMJ. 2020;370.

7. Yang R, Li X, Liu H, et al. Chest CT severity score: an imaging tool for assessing severe COVID-19. 2017;282(2):2017.

8. Zhang R, Tie X, Qi Z, et al. Diagnosis of COVID-19 Pneumonia Using Chest Radiography: Value of Artificial Intelligence. RSNA; 2020.

9. Yasin R, Gouda GW. Chest X-ray findings monitoring COVID-19 disease course and severity. Egypt J Radiol Nucl Med. 2020;51(1):1. doi:10.1186/s43055-020-00296-x

10. Borghesi A, Zigliani A, Masciullo R, et al. Radiographic severity index in COVID-19 pneumonia: relationship to age and sex in 783 Italian patients. Radiol Medica. 2020;125(5):461-464. doi:10.1007/ s11547-020-01202-1

11. Wong HYF, Lam HYS, Fong AHT, et al. Frequency and distribution of chest radiographic findings in patients positive for COVID-19. Radiology. 2020;296(2):E72-8. doi:10.1148/radiol.2020201160

12. Barnard C Age, comorbidities linked to poor COVID-19 outcomes in patients with rheumatic diseases. 2020. Available from: https://rheu matology.medicinematters.com/covid-19/infection/age-comorbidities -poor-outcomes-patients-rheumatic-diseases/18117812. Accessed May 13, 2021.

13. Borghesi A, Maroldi R. COVID-19 outbreak in Italy: experimental chest X-ray scoring system for quantifying and monitoring disease progression. Radiol Medica. 2020;125(5):509-513. doi:10.1007/ s11547-020-01200-3.

14. Warren MA, Zhao Z, Koyama T, et al. Severity scoring of lung oedema on the chest radiograph is associated with clinical outcomes in ARDS. Thorax. 2018;73(9):840-846. doi:10.1136/thoraxjnl-2017211280

15. Cozzi D, Albanesi M, Cavigli E, et al. Chest X-ray in new Coronavirus Disease 2019 (COVID-19) infection: findings and correlation with clinical outcome. Radiol Medica. 2020;125(8):730-737. doi:10.1007/s11547-020-01232-9

16. Wasilewski PG, Mruk B, Mazur S, Półtorak-Szymczak G, Sklinda K, Walecki J. COVID-19 severity scoring systems in radiological imaging - a review. Polish J Radiol. 2020;85(1):e361-8. doi:10.5114/ pjr.2020.98009

17. Signoroni A, Savardi M, Benini S, et al. End-to-end learning for semiquantitative rating of COVID-19 severity on Chest X-rays. 2020; Available from: http://arxiv.org/abs/2006.04603. Accessed May 13, 2021.

18. Hui TCH, Khoo HW, Young BE, et al. Clinical utility of chest radiography for severe COVID-19. Quant Imaging Med Surg. 2020;10(7):1540-1550. doi:10.21037/qims-20-642
International Journal of General Medicine

\section{Publish your work in this journal}

The International Journal of General Medicine is an international, peer-reviewed open-access journal that focuses on general and internal medicine, pathogenesis, epidemiology, diagnosis, monitoring and treatment protocols. The journal is characterized by the rapid reporting of reviews, original research and clinical studies across all disease areas. The manuscript management system is completely online and includes a very quick and fair peer-review system, which is all easy to use. Visit http://www.dovepress.com/ testimonials.php to read real quotes from published authors. 